\title{
ON THE RESISTANCE OF HUMAN ERYTHROCYTES TO COBRA VENOM.*
}

\author{
RICHARD WEII.
}

(From the Department of Experimenial Therapeutics, Cornell University Medical College, New York.)

IT was pointed out by the author ${ }^{\mathrm{r}}$ in a previous paper that the varying resistance of red cells to hemolytic agents is very probably to be regarded as an adaptive or protective mechanism. In the attempt to verify this hypothesis, a number of hemolytic agents have been tested against a series of human red cells derived from individuals suffering with different types of disease. Of these hemolytic agents, cobra venom ${ }^{2}$ gave results which seem to be of especial interest. Similar results, but much less striking in character, were obtained with saponin and digitonin. Rattlesnake venom has not been sufficiently tested to permit of a full report. In this connection, it was observed by H. Sachs ${ }^{3}$ that red cells taken from different individuals of the same species manifest a varying degree of resistance to the action of cobra venom, and he suggested that the venom might be used as an indicator of the amount of lecithin present in various tissues. Bang has recently corroborated this observation as to individual variations in resistance to cobra venom.

Method.-Blood is drawn with an aspirating syringe from one of the veins at the bend of the elbow, in amounts of not less than 10 c.c. Of this blood at least 2 c.c. is expelled into a test tube containing about the same quantity of a two per cent solution of sodium citrate; the remainder is kept and the serum tested according to the method of Wasserman or Noguchi. The corpuscles should not be prepared by shaking. The citrated plasma may be prepared for use on the same day, or may be kept as long as 48 hours in the ice box. It is centrifuged four times, the corpuscles being washed in a 0.9 per cent solution of common salt. In carrying out this process of washing, it is essential to remove the serum very thoroughly, inasmuch as it interferes very decidedly, even in slight traces, with the action of cobra venom. The washed cells arc made up into a four per cent suspension in 0.9 per cent common salt solution. The celis are centrifuged in very accurately graduated centrifuge tubes, and their accurate dilution is a matter of very great importance. The cell suspensions may be tested when fresh, or after a brief sojourn in the ice chest. If they are to be tested

* Received for publication November 12, 1909 .

Proc. Soc. Exper. Biol, and Med., Igog, 6, pp. 49-5r.

$=I$ am indebted to Drs. Flexner and Noguchi for samples of venom.

3 Kraus and Levaditi, Handbuch, Jena, 1907, I, pp. 254, 258. 


\section{Resistance of Human Erythrocytes to Cobra Venom 689}

immediately, the salt solution in which they are to be suspended should be kept in the ice chest until used; in this manner, the initial temperatures at which the mixtures of cobra venom and cells are made is always approximately the same. The cobra venom, which is kept in the dried condition, is made up very accurately in small quantities into a 0.05 per cent solution in 0.9 per cent solution of common salt. From this stock solution, higher dilutions are prepared as required, and are kept at a constant low temperature. The solutions of venom kept in this manner remain of a constant strength for several days at least. The resistance of the corpuscles to cobra venom may be tested roughly within an hour, or more delicately after 24 hours. If the rapid results are desired, venom solutions of $I$ in 8,000, and of $I$ in $x 5,000$ are prepared. If the results are to be read on the following day, four solutions are prepared, namely, $I$ in $10,000,20,000,30,000$, and 40,000 . One c.c. of the red cells is added to I c.c. of the venom solutions. If the rapid method is employed, the mixtures are incubated at $40^{\circ}$ for 30 minutes, and all cells which show no hemolysis in I to 8,000 after this time are considered positive, while all which show destruction at I to 15,000 are considered negative. If the second method is employed, and this is the more delicate of the two, the mixtures are incubated for one hour, after which a preliminary inspection will almost invariably reveal the final results of the tests. At this stage, cells showing no hemolysis with I to 10,000 venom are strongly positive, cells showing no hemolysis with I to 20,000 are positive, cells showing very slight hemolysis at I to 30,000 are weakly positive; cells showing moderate destruction at $\mathbf{I}$ to 30,000 are negative, and cells showing the least appreciable hemolysis at I to 40,000 are strongly negative. After a preliminary reading, the mixtures are again shaken, and are kept in the ice box over night, the final readings being made on the following morning. At the final reading, any cells which do not show complete hemolysis at $\mathbf{I}$ to $\mathrm{r}, 000$ are regarded as strongly positive; cells only moderately hemolyzed at I to 20,000 are regarded as positive; cells showing complete destruction at $\mathrm{I}$ to 30,000 are regarded as negative; cells showing complete destruction at I to 40,000 are regarded as extremely susceptible to the venom and are denoted in the abbreviated descriptions as "____." The meaning of the various degrees of hemolysis can easily be deduced from these data. The results should be absolutely concordant for the whole series of dilutions; if there appear to be discrepancies, the entire set of tubes should be rejected and the mixtures made afresh. The figures given above hold, of course, for the venom which formed the basis of these experiments; it is probable that other samples might differ in strength. At all events, a preliminary titration against corpuscles derived from cases of known lues and controls suffices to determine the values for any given sample. Inasmuch as the strength of the dried venom does not vary, this preliminary titration gives a constant standard, which is practically permanent, since one gram of venom suffices for 5,000 complete tests. It should furthermore be stated that the standardization is different for infants as compared with adults. It was long ago pointed out by Sachs that the resistance to various hemolytic agents, including venom, increases materially in the change from early to adult life in some of the lower species. The same condition holds true of man. In case of infants and young children, it is found that normal red cells hemolyze much more readily than those of adults. The cells, however, acquire the same degree of resistance as do those of adults in disease.

The cases which form the basis of the present communication number IgI. They were obtained from the wards of the German 
Hospital, from the neurological dispensary of the Presbyterian Hospital, through the kindness of Dr. Schlapp, and from the genitourinary and other departments of the Cornell University dispensary, through the kindness of Dr. Hastings and his assistants. The material was uniformly submitted to the procedures described above. The degree of hemolysis in the various tubes was described, according to the notation in common use, as Complete $(\mathrm{C})$, incomplete $(+++)$, very marked $(++)$, moderate $(+)$, slight (sl), and absent $(-)$. The interpretation of these degrees of hemolysis for the different concentrations of venom has been previously described. In interpreting the degree of resistance of the red cells, three independent criteria are employed, which, however, practically always vary in the same direction and to a similar extent, so that the practical gauging of the results is very much simpler and more accurate than in most hemolytic experiments. These criteria are: the rapidity with which hemolysis begins, the degrec of completeness at the end of the period of observation, and the tendency of the red cells, as the test proceeds, either to sink rapidly to the bottom of the tubes or to remain suspended in the fluid. In cases in which the red cells are susceptible, the hemolysis begins early, often within the first half-hour in the incubator; it tends to be marked in the moderate dilutions of venom at the end of the experiment; and the undestroyed red cells sink rapidly to the bottom of the tubes. These conditions are reversed in the case of resistant corpuscles. The cause of the change in the relative specific gravities of the red cells and the fluid has not been determined; it is certainly a very marked feature.

In studying the blood from a large number of human individuals, some of them normal, others afflicted with a variety of acute or chronic diseases, it is at once evident that there is a certain variability in the resistance of the red cells to the action of cobra venom. That this should be the case is not entirely surprising. It might a priori be expected that there would be some variability among tissue cells in this respect, as there is in all others. Indeed, it has been pointed out by a number of observers that such variability toward the action of other hemolysins than cobra venom exists among the individuals not only of the human but of other species. In one respect, however, namely, the very wide margin of difference between susceptible and 
resistant cells, variability toward cobra venom differs from that toward most other hemolysins. The condition which presents the most marked degree of resistance to the action of cobra venom is syphilis, after it has passed the primary stage. Normal individuals, and various diseased conditions manifest variability of resistance within certain narrow limits, but this never approaches the degree of resistance which is characteristic of syphilis. It is this fact, which makes it possible to construct a cobra spectrum, so to speak, with all the cases of syphilis grouped at one end and those of other conditions grouped in a graded series below it. On this account, also, it has been possible to group all cells which manifest resistance of a certain grade as positive, and all others which are distinctly less resistant as negative. Upon analysis it is found that all of the cells of the "positive" series are derived from advanced syphilitics, while all those of the "negative" series belong to other conditions.

In analyzing the results of the present series of tests, the diagnosis of cases has been made chiefly from the clinical history and findings. In all doubtful cases, the serum of the cases has also been tested according to the method of Wasserman and Noguchi. ${ }^{x}$ The results may be considered from various standpoints. Taking, first, the cases with a distinct history of syphilis, and marked evidences of an active syphilitic process, these are found to be uniformly marked by resistance to the cobra venom, and to fall into the group of positives. Of this type of cases, the series includes patients with syphilis of the skin, of the eye, of the central nervous system, of the aorta and great vessels, and of the bones and joints. The infection has ranged from several months to 2I years previously. In very few of these cases has a negative reaction been obtained. In almost all of them the Noguchi or Wasserman reactions were positive.

Then there is a considerable number of cases which have given a history of syphilitic infection, generally from five to forty years previously, who presented at the time of examination no evidences of an active syphilitic process. Some of these cases had been treated, and some had not. Of this group, the majority of the treated cases gave a negative reaction. Of the untreated cases, a considerable

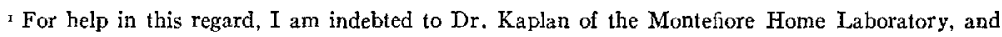
to Drs. Warren and Schwarz, of the Cornell Clinical Laboratory. Recently Dr. Noguchi has examined sera for me. 
number gave a positive reaction, which varied from a moderate to a marked degree. The results of the Wasserman and of the Noguchi reaction in this group of cases were not in complete accordance with the resistance reactions. In very few of the old, inactive, untreated cases, did they give a positive result. There is another group of cases in which there is no history of syphilitic infection, but in which, on clinical grounds, it secms necessary to infer a syphilitic origin of the disease. Of such cases, the present series includes such different conditions as habitual abortion, numerous stillbirths, hereditary infantile disease, certain bone diseases, certain types of cirrhosis of the liver, and certain nervous diseases such as tumor of the brain. This group excludes cases of tabes dorsalis. Of these cases, the majority were positive according to the cobra venom test, thus confirming the clinical diagnosis; they reacted, as a rule, in the same manner to the Wasserman method. In a very small number of cases, the Wasserman reaction was positive, while the venom test was negative; it is not yet possible to report on the true status of these cases.

In the cases taken as controls, it was twice possible to obtain a positive reaction. One of these individuals, who was suffering from a large cancer of the stomach, subsequently confessed to a syphilitic infection 20 years previously, which had again broken out i2 years later. The other, a case of Stokes-Adam's disease in the German Hospital, persistently denied syphilitic infection; in this case, the Wasserman and Noguchi tests were positive; the results of the therapeutic tests are not yet conclusive. Of the cases in which it was not possible to obtain a syphilitic history, but in which the clinical condition suggested syphilis, a very considerable proportion was constituted by the cases of locomotor ataxia. These gave a very high percentage of positive results with Wasserman reaction or the Noguchi modification, whereas a smaller number indicated a luetic origin with the cobra venom test.

The very early cases of lues, with chancre, or the first eruption, are often distinguished by the opposite condition. In them the cells are more than normally susceptible to the action of the venom. Frequently, at the end of one hour incubation, they show advanced hemolysis in the tubes containing I to 30,000 venom, and by the next 
day often all four tubes are completely hemolyzed. Within one to six months, however, from the appearance of the primary sore, the condition of increased resistance of the cells is manifest, and is apparently retained for years, unless abolished by treatment. It has not been possible to follow the development of this change of resistance in any individual case, owing to the obvious fact that cases are treated as soon as diagnosed. The above deductions are drawn from the observation of a series of early cases at various stages. The effect of treatment by means of mercury is gradually to abolish the resistance of the red cells. This effect, however, is only slowly accomplished. This evidence of syphilis, indeed, persists sometimes for weeks, or even months, after treatment has abolished the Wasserman reaction. The bearing of this fact on therapeutic procedures will be subsequently considered.

The non-syphilitic cases of this series have not, with the few questionable exceptions noted above, given any positive reactions. There have occasionally in this class been cases which verged on the positive reaction but none which could be unquestionably grouped as such. Among these have been cardio-nephritic, cancerous, and tuberculous cases; they have not as yet caused any actual confusion in the determinations. It should be stated, however, that the series is still too small to permit of a wide generalization excluding all non-syphilitic cases. For example, no typhoids or pneumonias had been tested.

There is a certain class of cases which has been found to give a positive Wasserman reaction, in spite of the fact that they are well known not to be syphilitic. These are, for example, cases of scarlet fever, leprosy, scleroderma, and some cases of polycythemia. Of scarlet fever six cases, of scleroderma one, and of polycythemia two, have been examined, and none of them has evinced an increased resistance of the red cells to cobra venom. In fact, the scarlet fever cases rather showed supersusceptibility to the venom.

Such is, in brief, a summary of the findings in the reactions done up to the present time. The results may be considered from either a practical or a purely theoretical standpoint. Practically, it would scem superfluous to provide another blood test for syphilis, when the Wasserman and the Noguchi reactions seem so completely to fulfil 
the needs of the clinic. On the other hand, in certain particulars, cobra venom might offer certain advantages over the Wasserman reaction, should it prove as reliable a criterion in a larger collection of cases as it has in the present series. These advantages appear to be the following: The method is much simpler and the labor much less. Less blood is required, so that a few drops from the lobe of the ear suffice for a reaction; this is of some importance in infants. Cases of scarlatina and of leprosy do not offer a source of confusion. The reaction is more marked in old, apparently dormant cases. The reaction persists much longer after mercurialization, thus offering a further diagnostic and therapeutic test. The reaction is possible in cases of jaundice, whereas the Wasserman is not. Aside from its practical application, the reaction suggests certain problems of a purely theoretical nature. It is apparently the first case of a constant and characteristic alteration in tissuc cells, as opposed to the serum, in response to a given pathological condition. The question is whether this alteration is to be regarded as a direct result of the action of the syphilitic virus, or as a reactive and protective response of the organism to the action of that virus. The former alternative suggests a comparison with the effects of the hypodermic injection of phenyl-hydrazin, which produces a rapid and striking increase in the resistance of the red blood cells to all hemolytic agents. On the other hand, there are certain features of the present case which seem to oppose such an interpretation. The preliminary period of hypersusceptibility would seem to indicate that the resistance cannot possibly be the result of the immediate action of the syphilitic virus on the red cells, but that it is a reactive phenomenon. In this connection, the author may recall the fact that about a year ago he published a somewhat similar observation drawn from experimental data. It was found that repeated hypodermic injections of saponin over a long period of time in rabbits results in the production of red blood cells which possess a heightened degree of resistance to saponin, but to no other hemolytic agents; this period of increased resistance is, however, preceded by a short but distinct period of hypersusceptibility to the poison. It was suggested at the time that the hypersusceptible cells represented the first generations of red cells which had come into contact with the poison; that they had been partially 
charged with it, or partially injured by it, and were therefore more easily destroyed. The later and resistant generations were believed to represent a reactive process. It is not difficult to conceive of some such process in the case of saponin, but it is certainly a very different matter to explain the action of syphilis in producing an increased resistance to so different a poison as cobra venom. It has been shown by repcated experiments that this resistance to cobra venom is specific, and that the syphilitic red cells show no increase of resistance to other hemolytic agents. It is perhaps permissible to suggest that the luetic virus attacks the same cellular constituent as does cobra venom. In the discussion of this possibility, one is forced unfortunately to rely on a considerable number of only partially tested data, and the deductions are likely to be correspondingly inaccurate and unreliable. It has been found by Kyes and Sachs that cobra venom unites with lecithin to form a toxic cobra-lecithid. They also showed that this lecithin might be derived from the red blood cells themselves, where it was present as an "endocomplement." In conformity with this view, it would be possible to conceive that the amount of lecithin in the red cells had been diminished in luetic conditions, thus leading to the production of a smaller amount of the toxic cobra-lecithid in the red cells. That lues does in fact attack the lipoids of the body, and that the amount of lecithin which can be extracted from the tissues is less in syphilitic conditions than in normal individuals, is apparently well attested. On the other hand, it is possible, as has been personally suggested to me by Noguchi, that the sodium citrate plays an essential rôle in the reaction, as it has been shown to do in cobra hemolysis. This possibility is supported by the fact that skaken cells fail to exhibit the differences above described. Which, if any, of these possibilities represents the correct interpretation of the phenomena, can be determined only by further study of the conditions. 